\title{
Spinal cord ischaemia complicating meningococcal meningitis
}

\author{
SONIA S. SWART \\ M.B., M.R.C.P. (UK)
}

IAN F. PYE

M.B., M.R.C.P. (UK)

Department of Neurology, Leicester Royal Infirmary, Leicester LE1 5WW

\begin{abstract}
Summary
An extensive ischaemic cord syndrome developed in a patient with meningococcal meningitis complicated by 2 respiratory arrests but not by any period of prolonged hypotension or other signs of cardiovascular collapse. Excellent functional recovery occurred after intensive rehabilitation.
\end{abstract}

\section{Case history}

A 15-year-old schoolboy presented with one day's history of severe headache and progressive confusion following several days of 'flu-like illness. The clinical findings on admission were a temperature of $40^{\circ} \mathrm{C}$, marked neck stiffness, a depressed conscious level with response to pain only, and a generalized erythematous macular rash over the arms and trunk. Lumbar puncture confirmed purulent meningitis due to Neisseria meningitidis.

He was treated with intravenous benzyl penicillin and probenecid. Within $7 \mathrm{hr}$ he was responding to command, drinking unaided, and his temperature had fallen to $38.7^{\circ} \mathrm{C}$. At this point he had a respiratory arrest but intubation was followed by the immediate return of spontaneous respiration and recovery of his pre-arrest state. Twelve hours later after further respiratory arrest he required assisted ventilation and did not regain consciousness for several hours. Neither arrest was accompanied by hypotension. After the second episode, it was noted that he was unable to move. The neurological findings were as follows: he was alert and well orientated and able to mouth words; there was no attempt at spontaneous respiration; neck stiffness remained marked; the visual axes diverged at rest, although external eye movements were full, and there was nystagmus on lateral gaze bilaterally; facial sensation to light touch and pin-prick was impaired bilaterally and the corneal reflexes were sluggish; visual fields and fundi were normal; the gag reflex was absent but function of the 7th and 12th cranial nerves was normal and hearing was intact; he was unable to shrug his shoulders or turn his head, had a flaccid quadraplegia and was areflexic with absent plantar responses; sensation was normal in the upper limbs; there was altered light touch and pin-prick below $\mathrm{T} 4$ with absent position sense in the feet but preservation of vibration sensation; he developed urinary retention and required an indwelling catheter.

Tracheostomy was performed and the patient was ventilated. Four days later the conjugate gaze defect and nystagmus had improved and sensation to light touch and pin-prick was returning in the feet. Artificial ventilation was discontinued after 2 weeks. Neurological recovery proceeded in the following sequence: motor power on the left side and sensation on the right returned simultaneously; pin-prick and light touch sensibility recovered before temperature appreciation; the tendon reflexes returned slowly as did some power on the right and sensation on the left. Four weeks after admission he was transferred to a spinal unit for rehabilitation. At that time there was wasting and flaccid weakness of all 4 limbs, more marked distally and worse on the right. The supinator and knee jerks were absent bilaterally but the other tendon reflexes had returned and both plantar responses were extensor. The appreciation of light touch, vibration and joint position sensation had recovered but pin-prick and temperature sensibility remained impaired in the left leg. Eight weeks later he could walk holding parallel bars and 5 months later he was walking unaided. When reviewed 11 months after the illness he was able to jog and had no functional disability in the arms. There were some residual neurological signs consisting of minimal wasting of the intrinsic muscles of the left hand with slight weakness but normal power above the wrist. Formal testing of power in the legs was normal and he was able to walk on tiptoes but there was slight impairment of heel walking and heel toe walking. There was minimal clumsiness of alternate rapid movements in the right arm and leg and minimal right hyperreflexia. Both plantars were extensor and the abdominal reflexes were absent. Sensation 
was intact in the limbs apart from some contact dysaesthesia over the soles of the feet.

\section{Discussion}

The neurological signs following the respiratory arrest were compatible with an ischaemic lesion at the cervico-medullary junction plus patchy involvement of the brainstem rostrally. Within a few days the brainstem signs had resolved and the clinical picture suggested an infarct of the upper cervical cord in the anterior spinal artery territory. This was considered to be secondary to an infective arteritis, and although hypoxia may have contributed to the neurological deficit, it seemed more likely that the respiratory arrest itself was the result of involvement of the respiratory centres in the brainstem since on each occasion he was resuscitated immediately without any period of hypotension. The presence of transient signs of brainstem ischaemia may indicate occlusion of the anterior spinal artery close to its origins from the vertebral arteries.

Ischaemia of the cord, particularly in the vulnerable territory of the anterior spinal artery, has been noted in a variety of clinical situations. Early reports focused on syphilitic arteritis as a cause of the syndrome of anterior spinal artery occlusion (Spiller, 1909). More recent reviews emphasize the importance of atheromatous vessels (Henson and Parsons, 1967) with or without a period of prolonged hypotension as an aetiological factor (Silver and Buxton, 1974). Hughes (1978) describes spinal cord infarction as an unusual but important complication of Pott's disease and thrombosis of the radicular artery may occur in herpes zoster. Although ischaemic cord damage secondary to small vessel endarteritis may occur in pyogenic meningitis, reports of extensive infarction of the cord have not been found on reviewing the literature.

This case is reported for 2 reasons, firstly as an unusual complication of bacterial meningitis, and secondly, as a reminder that a young previously fit subject may have a remarkable potential for neurological recovery from a catastrophic situation.

\section{Acknowledgment}

We should like to thank Dr C. P. Alexander for referring the patient.

\section{References}

Henson, R.A. \& Parsons, H. (1967) Ischaemic lesions of the spinal cord: an illustrated review. Quarterly Journal of $\vec{\omega}$ Medicine, 36, 205.

Hughes, J.T. (1978) Pathology of the Spinal Cord, 2nd edn, pp. 110, 141. Lloyd-Luke, London.

Silver, J.R. \& BuXtoN, P.H. (1974) Spinal stroke. Brain, 97, 539.

SPILleR, W.G. (1909) Thrombosis of the cervical anterif median spinal artery: syphilitic acute anterior polio myelitis. Journal of Nervous and Mental Disorders, 36, 606 